\title{
Protein tyrosine phosphatase inhibition aids metabolically impaired progenitor stem cells
}

Katarzyna Kornicka-Garbowska

Lynda Bourebaba

Michael Röcken

Krzysztof Marycz

\section{Video Byte}

Keywords: progenitor stem cells, adipogenesis, protein tyrosine phosphatase 1B, PTP1B, low-molecularweight protein tyrosine phosphatase, LMPTP, mitochondria, protein tyrosine phosphatase, adipogenic differentiation, mitochondrial dynamics, mitochondrial bioenergetics, metabolic impairment, oxidative stress, antioxidant, oxidative phosphorylation, in vitro, cell culture, mitochondrial fusion, protein tyrosine phosphatase inhibitor, Cell Communication and Signaling

Posted Date: March 1st, 2022

DOI: https://doi.org/10.21203/rs.3.rs-1405888/v1

License: (9) This work is licensed under a Creative Commons Attribution 4.0 International License. Read Full License 


\section{Abstract}

Metabolic syndrome is becoming increasingly common among humans and domestic animals and is thought to be linked to dysfunction in adipose tissue components, including adipose progenitor stem cells (ASCs). The proteins PTP1B and LMPTP have been implicated in the development of metabolic disorders, but their roles in adipogenic differentiation of ASCs and modulation of mitochondrial dynamics in these cells remain unclear. To clarify this issue, a recent study treated ASCs from metabolically impaired horses with PTP1B and LMPTP inhibitors in vitro. Both selective inhibitors enhanced the differentiation of ASCs into adipose cells and increased the expression of PPARY, a master adipogenesis regulator, while the LMPTP inhibitor increased the expression of adiponectin, which helps protect against metabolic disorders. The compounds also improved antioxidant defense and altered mitochondrial bioenergetics. Finally, the inhibitors activated oxidative phosphorylation and enhanced mitochondrial fusion, contributing to enhanced adipogenesis. Although additional research is needed, the results show that PTP1B and LMPTP inhibitors can improve the ability of dysfunctional ASCs to differentiate and may aid in the development of treatments for insulin resistance and related metabolic conditions. 